Génét. Sél. Evol., 1986, 18 (3), 321-332

\title{
Méthode simplifiée \\ de calcul des valeurs génétiques des femelles tenant compte de toutes les parentés
}

\author{
J.P. POIVEY
}

INRA, Station d'Amélioration génétique des Animaux, B.P. 27, F 31326 Castanet Tolosan Cedex

\begin{abstract}
Résumé
La méthode proposée suppose déjà existantes les solutions concernant les effets fixes et les valeurs génétiques des mâles dans un modèle animal. Elle utilise les résultats de Henderson (1976) concernant le calcul de l'inverse de la matrice de parenté entre les individus dans une population non consanguine et l'application de la résolution des équations du modèle mixte, alliée à une triangularisation de la matrice afférente aux valeurs génétiques des femelles aboutit à la construction d'un algorithme de calcul très rapide.
\end{abstract}

Mots clés : Valeurs génétiques, parenté, BLUP modèle mixte, triangularisation.

\author{
Summary \\ Simplified computing method for estimation of the \\ breeding values of females with a relationship matrix
}

This method is based on the existence of estimates of fixed effects and male breeding values in an animal model. The inverse of the relationship matrix in a non-inbred population is calculated by the method of HENDERSON (1976), and the solution of the mixed model equations with a triangularisation of the matrix provides a very fast computing algorithm.

Key words : Breeding values, relationships, BLUP, mixed model, triangularisation.

\section{Introduction}

L'estimation des valeurs génétiques des animaux par la méthode du BLUP (Best Linear Unbiased Predictor) (HENDERson, 1973 et 1975) conduit généralement à la construction de matrices dont la taille devient souvent incompatible avec la capacité des ordinateurs les plus puissants et interdisent l'utilisation de microordinateurs, dont l'usage est pourtant de plus en plus répandu. Cette caractéristique propre à la méthode a limité son usage essentiellement à l'estimation des valeurs 
génétiques des mâles reproducteurs jugés sur descendance (modèle père) et son application à l'estimation des valeurs de l'ensemble des animaux, y compris les femelles et les descendants (modèle animal) a toujours été jugée très onéreuse lorsque l'on s'intéresse à des effectifs importants.

En France, dans l'espèce ovine, sont calculées les valeurs génétiques des brebis reproductrices pour leur valeur laitière et leur prolifícité. Ces calculs concernent environ 300000 têtes inscrites au contrôle de performances en fermes. On dispose de peu d'informations par individu ( 1 à 3 performances) et les caractères possèdent une faible héritabilité : 0,06 pour la prolificité (BodIN, 1979). Dans cette situation, l'amélioration de la précision des estimations des valeurs génétiques ne peut se faire qu'en prenant en compte le maximum d'informations disponibles sur les autres femelles apparentées à chaque candidat à la sélection.

Le but de la méthode proposée est d'éviter le calcul et l'inversion d'une matrice de taille importante et de fournir une méthode de résolution rapide du système d'équations concernant les femelles dans le cas où l'on connaît déjà une estimation des effets fixes (effets troupeaux, saisons, etc.) et les prédictions des valeurs génétiques des pères de ces brebis. L'obtention de ces 2 dernières séries de données sera également discutée.

\section{Méthode}

Pour décrire la k-ème performance $Y_{\hat{i k}}$ d'un animal $i$, on utilise le modèle suivant :

$$
Y_{i k}=X_{i k} \beta+Z_{i k} G+Z_{i k} P+E_{i k}
$$

qui correspond à un "modèle animal » où l'on fait les hypothèses suivantes :

$\beta$ est un vecteur d'effets fixes inconnus

$\mathrm{X}$ est une matrice d'incidence connue décrivant la répartition des effets fixes dans le dispositif expérimental, où $\mathrm{X}_{\mathrm{ik}}$ est une ligne correspondant à la k-ème observation de l'animal i

$\mathrm{G}$ est le vecteur des valeurs génétiques additives des $\mathrm{m}$ individus mesurés, dont la variance est connue et égale à $\mathrm{Ao}_{\mathrm{a}}^{2}$ (A matrice des coefficients de parenté entre les individus)

P est le vecteur d'un effet aléatoire lié à l'environnement permanent dans lequel sont exprimées les performances de l'individu i et dont la variance est supposée égale a $\sigma_{p}^{2}$

$\mathrm{Z}$ est la matrice d'incidence fournissant l'appartenance des $\mathrm{n}$ observations aux $\mathrm{m}$ individus

E est la variable aléatoire résiduelle de variance $\sigma_{\mathrm{e}}^{2}$.

On définit en outre la répétabilité du caractère par

$$
r=\frac{\sigma_{\mathfrak{a}}^{2}+\sigma_{\mathrm{p}}^{2}}{\sigma_{\mathrm{y}}^{2}}=\frac{\sigma_{\mathrm{a}}^{2}+\sigma_{\mathrm{p}}^{2}}{\sigma_{\mathrm{a}}^{2}+\sigma_{\mathrm{p}}^{2}+\sigma_{\mathrm{e}}^{2}}
$$


et son héritabilité par

$$
h^{2}=\frac{\sigma_{\mathrm{a}}^{2}}{\sigma_{y}^{2}}=\frac{\sigma_{\mathrm{a}}^{2}}{\sigma_{\mathrm{a}}^{2}+\sigma_{\mathrm{p}}^{2}+\sigma_{\mathrm{e}}^{2}}
$$

supposées identiques quel que soit le rang de la performance dans la carrière de l'animal.

Nous nous plaçons dans le cas bien particulier où nous possédons déjà une estimation des effets fixes $\widehat{\beta}$ qui étaient inconnus au départ.

Nous discuterons ultérieurement l'éventualité et la fréquence d'une telle situation. Les valeurs génétiques sont alors obtenues par la résolution des équations du modèle mixte (HENDERSON, 1975) :

où

$$
\left(\begin{array}{cc}
Z^{\prime} Z+A^{-1} \alpha \quad Z^{\prime} Z \\
Z^{\prime} Z & Z^{\prime} Z+I_{m} \gamma
\end{array}\right)\left(\begin{array}{c}
\widehat{G} \\
\widehat{P}
\end{array}\right)=\left(\begin{array}{l}
Z^{\prime}(Y-X \widehat{\beta}) \\
Z^{\prime}(Y-X \widehat{\beta})
\end{array}\right)
$$

$$
\begin{aligned}
& \alpha=\frac{\sigma_{\mathrm{e}}^{2}}{\sigma_{\mathrm{a}}^{2}}=\frac{1-\mathrm{r}}{\mathrm{h}^{2}} \\
& \gamma=\frac{\sigma_{\mathrm{e}}^{2}}{\sigma_{\mathrm{p}}^{2}}=\frac{1-\mathrm{r}}{\mathrm{r}-\mathrm{h}^{2}}
\end{aligned}
$$

On démontre facilement que, par absorption des effets aléatoires $P$, ce système peut s'écrire sous la forme suivante :

$$
\left(Z^{\prime} \mathrm{DZ}+\mathrm{A}^{-1} \alpha\right) \widehat{\mathrm{G}}=\mathrm{Z}^{\prime} \mathrm{D}(\mathrm{X}-\mathrm{X} \widehat{\beta})
$$

où $\mathrm{D}$ est une matrice carrée diagonale dont l'élément correspondant à un individu i est égal à

$$
\mathrm{d}_{\mathrm{i}}=\frac{\gamma}{\mathrm{n}_{1}+\gamma}
$$

$\mathbf{n}_{\mathbf{i}}$ représentant le nombre de performances observées pour cet animal.

Dans le cas d'une population non consanguine, le calcul de la matrice inverse des coefficients de parenté $A^{-1}$ peut s'effectuer de façon itérative selon des règles très simples démontrées par HENDERson (1976) et qui ont été résumées par Poutous (1980).

Pour chaque individu, auquel on attribue la place $\mathrm{i}$ dans la matrice et dont les parents connus sont codifiés $\mathrm{p}$ et $\mathrm{p}^{\prime}$, on ajoute successivement :

- $\Delta$ à l'élément (i,i) 
- $\Delta / 4$ aux éléments (p,p), (p, $\left.\mathrm{p}^{\prime}\right),\left(\mathrm{p}^{\prime}, \mathrm{p}\right)$ et $\left(\mathrm{p}^{\prime}, \mathrm{p}^{\prime}\right)$

- $-\Delta / 2$ aux éléments $(i, p),\left(i, p^{\prime}\right),(p, i)$ et $\left(p^{\prime}, i\right)$

Suivant la situation de parenté de l'individu i, $\Delta$ prend les valeurs :

- 2 lorsque les 2 parents sont connus

- 4/3 lorsque un seul parent est connu

- 1 lorsque les 2 parents sont inconnus.

Ce résultat se démontre aisément en construisant d'une manière récurrente les matrices $A$ et $A^{-1}$ : en ajoutant un nouvel individu, on vérifie le résultat du produit des 2 matrices (Poutous, 1980).

On remarque que les seuls éléments hors-diagonaux non nuls de la matrice $A^{-1}$ sont ceux correspondant aux places parents-produit et père-mère. Les équations associées aux mâles de la population peuvent donc être facilement éliminées en adaptant les règles de calcul à notre système d'équations :

- en considérant l'ensemble des mâles $h$ dont la mère est connue $f$, on soustrait des termes de droite d'indice f la quantité égale $-\left(\Delta_{h} \alpha\right) / 2$ multipliée par l'estimation de la valeur génétique de ce fils, ainsi que $\left(\Delta_{\mathrm{h}} \alpha\right) / 4=\alpha / 2$ multiplié par la valeur du père de $h$ si celui-ci est connu, puis on ajoute au terme diagonal $f$ correspondant de la matrice de parenté $\Delta_{\mathrm{h}} \alpha / 4$;

- ensuite, considérant l'ensemble des femelles, lorsque le père est connu, on retranche aux termes de droite du système les quantités égales à sa valeur multipliées $\operatorname{par}-\Delta \alpha / 2$ à l'élément correspondant à l'individu, et par $(\Delta \alpha) / 4=\alpha / 2$ à l'élément associé à la mère si celle-ci est également connue ;

- on applique les premières règles aux seuls éléments correspondant à l'individu et à la mère, à l'exclusion de ceux afférant aux pères qui ne figurent pas dans notre système d'équations.

Il est cependant important de noter que cette méthode simple ne fournit pas exactement $A^{-1}$, mais une matrice $A_{0}^{-1}$ qui concerne l'ensemble des $m$ animaux mesurés, augmentés de leurs $\mathrm{m}_{0}$ parents non mesurés et considérés comme base génétique de la population à condition d'étendre les règles de calcul à ces derniers. Généralement, plutôt que de rechercher exactement $\mathrm{A}^{-1}$, on préfère résoudre un système de dimension supérieure :

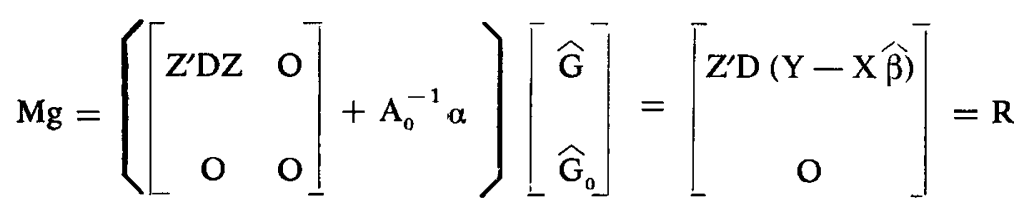

Le principe de notre methode repose ensuite sur la triangularisation de la matrice $\mathbf{M}$, c'est-à-dire la recherche d'une matrice triangulaire inférieure $L$ telle que $L L^{\mathbf{t}}=\mathbf{M}$.

Dans le cas d'existence de $\mathrm{L}$, la résolution du système $\mathrm{Mg}=\mathrm{R}$ se traduit par celle des 2 suivants (GASTINEL, 1966)

$$
\left\{\begin{array}{l}
\mathrm{Lh}=\mathrm{R} \\
\mathrm{L}^{\mathrm{t}} \mathrm{g}=\mathrm{h}
\end{array}\right.
$$


Or la solution d'un tel système est très simple, puisque :

$$
\left\{\begin{aligned}
\mathrm{h}_{1} & =\frac{\mathrm{r}_{1}}{\mathrm{l}_{11}} \\
\vdots & \\
\vdots & 1 \\
\mathrm{~h}_{\mathrm{i}} & =\frac{1}{\mathrm{l}_{\mathrm{ii}}}\left(\mathrm{r}_{\mathrm{i}}-\sum_{\mathrm{j}=1}^{\mathrm{i}-1} \mathrm{l}_{\mathrm{ij}} \mathrm{h}_{\mathrm{j}}\right)
\end{aligned}\right.
$$

Si l'on triangularise $M$ suivant la méthode de CHOLESKY, on a :

$$
\begin{aligned}
l_{i i} & =\sqrt{m_{i i}-\sum_{k=1}^{i-1} 1_{k i}^{2}} \\
l_{j 1} & =\frac{m_{j i}-\sum_{k=1}^{i-1} 1_{j k} l_{i k}}{1_{i 1}}
\end{aligned}
$$

pour $\mathrm{j}>\mathrm{i}$

Sachant que les seuls éléments hors-diagonaux non nuls sont les intersections fille-mère, cette procédure de calcul s'opère facilement en se déplaçant dans le sens des lignes de la matrice. Si celle-ci est organisée de telle façon que les filles précèdent les mères, on constate que, dans le calcul de $l_{\mathrm{ji}}$, interviennent des éléments de la forme $l_{\mathrm{jk}} l_{\mathrm{ik}}$ pour $\mathrm{k}<\mathrm{i}$ et correspondant aux cas « $\mathrm{k}$ fille de $\mathrm{j}$ » et « $\mathrm{k}$ fille de $\mathrm{i}$ ». Ces 2 événements étant incompatibles, et $m_{\mathrm{ji}}$ étant égal à $-(\Delta \alpha) / 2$ dans le cas où $\mathrm{j}$ est fille de $i$, nul dans le cas contraire, on montre aisément par récurrence que les éléments nuls de $M$ restent nuls dans $L$.

On a alors :

$$
1_{i i}=\sqrt{\frac{n_{i} \gamma}{n_{i}+\gamma}+\Delta_{i} \alpha+S_{i}}
$$

et

$$
1_{\mathrm{ji}}=-\frac{\Delta_{\mathrm{i}} \alpha}{21_{\mathrm{ii}}} \quad \text { pour } \mathrm{j} \text { mère de } \mathrm{i}
$$

avec $S_{i}=\sum_{j \text { filles de } i}\left(\left(\Delta_{j} \alpha\right) / 4-1_{i j}^{2}\right)+\sum_{j \text { fils de } i}\left(\Delta_{j} \alpha\right) / 4$

On peut vérifier que $M$ est définie positive, propriété équivalente à l'existence de L (GASTINEL, 1966).

Une condition suffisante est que $S_{i} \geqslant O$, propriété aisément démontrée par récurrence, puisque si $S_{j} \geqslant O$, alors

$$
\begin{gathered}
\left(\Delta_{\mathrm{j}} \alpha\right) / 4-\underset{\mathrm{I}_{\mathrm{j}}}{2} \geqslant\left(\Delta_{\mathrm{j}} \alpha\right) / 4-\frac{\left(\left(-\Delta_{\mathrm{j}} \alpha\right) / 2\right)^{2}}{\Delta_{\mathrm{j}} \alpha} \\
\geqslant 0
\end{gathered}
$$




\section{Discussion}

La méthode de calcul proposée permet un gain de place en mémoire très important. Il suffit en effet de 2 tableaux réels à une seule dimension et de longueur égale au nombre de femelles $\mathrm{n}$, pour le calcul des éléments non nuls de la matrice triangularisée, alors qu'une méthode classique, construisant l'inverse de la matrice de parenté stockée sous forme triangulaire utiliserait un tableau réel de dimension $(n+1) n / 2$. Si $p$ est le nombre de mâles, le nombre d'opérations sera au maximum de :

- $\mathrm{n} \quad$ racines carrées

$-7 n+2 p$ addition ou soustractions

- $10 \mathrm{n} \quad$ multiplications ou divisions.

Lorsque l'on recourt à des inversions directes de matrices, le nombre d'opérations devient une fonction de $\mathrm{n}^{3}$ et $\mathrm{n}^{2}$.

Cependant, il est bien évident que cet algorithme ne constitue qu'une phase calculatoire dans la résolution d'un système où il faut également estimer la valeur génétique des mâles et des effets fixes du modèle présenté initialement.

Pour cela on peut tout d'abord utiliser un modèle père ou un modèle père et mère, dans lequel la performance d'un individu est décomposée en effets aléatoires liés à la transmission mendélienne des caractères par les parents. De très nombreuses études ont porté sur ces modèles simplifiés qui, pour leurs estimations, ne peuvent cependant pas prendre en compte l'ensemble de toutes les parentés que nous avons utilisées. BONAITI \& BRIEND (1986) soulignent, cependant, que même dans un modèle père l'estimation simultanée des effets élevages et des effets pères devient impossible par résolution directe et qu'il est nécessaire de recourir à des méthodes itératives comme celle de Gauss-SEIDEl. Ainsi que de nombreux autres auteurs, ils remarquent que l'efficacité d'une telle méthode est accrue lorsqu'elle procède par blocs, en résolvant par exemple successivement des systèmes liés à chacun des effets du modèle : effets fixes, valeurs génétiques des mâles, valeurs génétiques des femelles, etc.

Lorsque l'on ne connaît pas d'effets fixes et que l'on ne possède pas d'estimées des valeurs génétiques des mâles, on note que la résolution du modèle (1) par une méthode itérative par blocs conduit à la résolution de notre équation (2) pour les valeurs génétiques. La ségrégation de ces dernières entre mâles et femelles autorise l'adoption de nos règles de calcul pour la résolution du bloc associé aux femelles. Les mêmes règles pouvant être transposées à l'estimation des valeurs génétiques des mâles, on constate que l'on possède ainsi un moyen de calcul très rapide qui permet d'envisager la résolution des systèmes les plus généraux et en particulier un "modèle animal ", tout en restant dans le cadre de la méthodologie du BLUP.

Lorsque l'on ne considère qu'une seule performance par animal, la matrice des effets toupeau $X$ année $\times$ saison est diagonale et le système lié à ces effets se résout très facilement. Mais si l'on considère des performances multiples produites à des moments différents, l'absorption des effets aléatoires liés à l'environnement permanent $\mathbf{P}$ fait apparaître entre les différentes modalités, des termes extra-diagonaux de la forme $\sum_{1}-\frac{1}{n_{1}+\gamma}$. 
On remarquera en outre que, dans un processus de calcul itératif, et ceci pour chaque phase calculatoire liée au type d'effet estimé, l'établissement des termes de gauche du système, et l'inversion de la matrice, pour les effets fixes, ne sont effectués qu'une seule fois et qu'à chaque nouvelle itération on ne recalcule que les termes de droite.

Notre méthode calculatoire peut être appliquée intra-troupeau, dans la logique de la stratégie envisagée par Henderson (1975) qui limite la taille des matrices à inverser. Mais, appliquée ainsi, elle n'autorise pas' la considération d'échanges de femelles entre élevages et conduit à négliger à la fois certains liens de parenté entre femelles et des performances d'un même individu exprimées à des endroits différents. Le fait de réduire les tailles des tableaux et le nombre d'opérations à des valeurs proportionnelles au nombre d'individus (et non à leur carré) laisse clairement entrevoir une application du calcul à l'ensemble de la population.

Si la méthode de Christensen (1981) fournit également des moyens de calcul rapides, en revanche elle est difficilement comparable au BLUP et elle ne considère que l'information obtenue sur ascendance alors que nous utilisons la totalité de l'information généalogique.

Le modèle (1) qui a servi de base à notre étude peut connaître des variations selon les cas étudiés. On peut en particulier considérer que les variances résiduelies ne sont plus indépendantes du rang de la performance : $\operatorname{Var}(E)=R \underset{\sigma_{e}}{2}$ où $R$ est une matrice carrée diagonale dont chaque élément est égal à une valeur qui ne dépend que du rang de la performance. Alors le système d'équation (2) doit être remplacé par

$$
\left[\begin{array}{cc}
Z^{\prime} R^{-1} Z+A^{-1} \alpha & Z^{\prime} R^{-1} Z \\
Z^{\prime} R^{-1} Z & Z^{\prime} R^{-1} Z+I_{m} \gamma
\end{array}\right]\left[\begin{array}{c}
\widehat{G} \\
\widehat{P}
\end{array}\right]=\left[\begin{array}{l}
Z^{\prime} R^{-1}(Y-X \widehat{\beta}) \\
Z^{\prime} R^{-1}(Y-X \widehat{\beta})
\end{array}\right]
$$

les éléments $d_{i}$, obtenus par absorption des effets de l'environnement permanent liés à l'individu, deviennent :

et la formule (3) s'écrit :

$$
\mathrm{d}_{\mathrm{i}}=\frac{\gamma}{\gamma+\sum_{\mathrm{k}} \frac{1}{\mathrm{r}_{\mathrm{k}}}}
$$

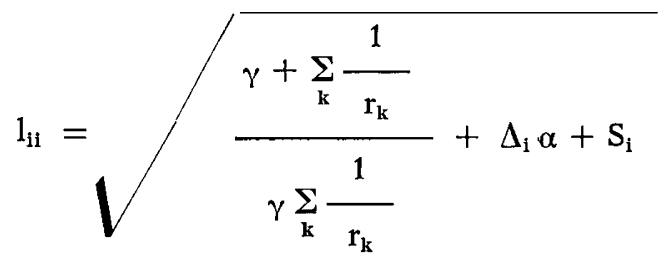

Si R est bloc-diagonale, un bloc correspondant à chaque individu, il est clair que l'absorption nécessite une inversion de matrice pour chaque individu, mais que notre méthode peut ensuite être facilement adaptée au moment de la somme de la 
matrice d'incidence des moindres carrés où les effets de l'environnement permanent sont absorbés, avec l'inverse de la matrice de parenté.

Si l'on introduit dans le modèle des effets aléatoires associés à un environnement maternel commun, nos règles de calcul ne peuvent les prendre directement en compte, car on doit considérer des covariances environnementales entre demi-sœurs. Il est bien certain, cependant, que l'on peut alors recourir à une méthode de résolution bloc-itérative, qui dissocie les estimations des effets maternels de celle des valeurs génétiques pour laquelle notre méthode reste valable.

Une autre utilisation possible de cet organigramme peut se situer au niveau des élevages où il est souhaitable de fournir des résultats très rapidement. Dès la saisie des données dans l'élevage, un micro-ordinateur qui contiendrait la valeur d'effets pères, d'effets saisons et autres effets fixes calculés sur un site national au cours des campagnes précédentes, permettrait de donner une première approximation des valeurs génétiques des femelles permettant d'attendre la réactualisation des effets pères, le calcul d'effets troupeau $X$ année $\times$ saison et le calcul définitif des index femelles sur le site central.

\section{Conclusion}

La méthode de calcul proposée pour l'estimation des valeurs génétiques des femelles autorise une meilleure utilisation, en temps et capacité, des moyens informatiques disponibles. Elle permet en outre d'améliorer les modèles utilisés en considérant davantage d'informations sur les parentés des individus. En particulier, intégrée dans une méthode plus générale de calcul itérative par blocs, elle laisse envisager la mise en œuvre des modèles les plus complets (modèle animal) qui n'étaient, jusqu'à présent, réservés qu'à des échantillons restreints d'animaux.

Reç le 3 juillet 1985.

Accepté le 21 mars 1986.

\section{Références bibliographiques}

Bodin L., 1979. Estimation des paramètres génétiques de la taille de portée des agnelles Lacaune après fécondation sur oestrus naturel et induit. Ann. Génét. Sél. Anim., 11 (4), 413-424.

BonaIti B., BRIEND M., 1986. Computing algorithm for dairy sire evaluation on several lactations considered as the same trait. Génét. Sél. Evol., 18 (1), 41-54.

Christensen L.G., 1981. Basic principles in the estimation of breeding values by direct updating. Z.' Tierzücht. Züchtungsbiol., 98, 131-150.

Gastinel N., 1966. Analyse numérique linéaire. 364 pp., Hermann, Paris.

Henderson C.R., 1973. Sire evaluation and genetic trends. In : Proc. Anim. Breeding Genet. Symp. in Honor of Dr. Jay L. Lush., 10-41. Amer. Soc. Anim. Sci. and Amer. Dairy Sci. Assoc, Champaign, Illinois. 
HENDERSON C.R., 1975. Use of all relatives in intra-herd prediction of breeding values and producing abilities. J. Dairy Sci., 58 (12), 1910-1921.

HENDERSON C.R., 1976. A simple method for computing the inverse of a numerator relationship matrix used in prediction of breeding values. Biometrics, 32, 69-83.

Poutous M., 1980. Note sur l'inverse d'une matrice de parenté. Ann. Génét. Sél. Anim., $12(2), 215-218$.

\section{Annexe \\ Construction de l'algorithme de calcul}

\section{A. Préparation des données}

Cette phase consiste dans le calcul des effets fixes du modèle et des valeurs génétiques des mâles (discutées ultérieurement). On constitue un fichier de données, trié dans l'ordre allant des filles vers les mères, dont chaque enregistrement contient la codification $i$ de la femelle, $j$ et $k$ celles de sa mère et de son père, le nombre $n_{1}$ de performances et la somme de ces performances $r_{i}$ corrigées des effets fixes. Le

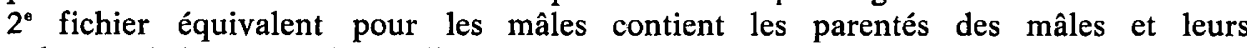
valeurs génétiques individuelles estimées GENE qui seront stockées ultérieurement dans un tableau VAL. Les fichiers doivent être complétés par la base génétique de la population, c'est-à-dire les parents non mesurés pour lesquels $n_{i}, r_{i}, j$ et $k$ sont nuls.

\section{B. Triangularisation de la matrice et calcul des termes de droite}

Un tableau TAB est nécessaire pour stocker les éléments diagonaux de la matrice, alors qu'un second, PAR, de même dimension est réservé pour les éléments extradiagonaux correspondant, pour un individu au seul élément non nul qui le lie à sa mère. Un troisième tableau MERE contient les codifications des mères de chaque animal et les termes de droite sont mis dans RHS.

Dans un premier temps, l'exploitation du fichier des mâles permet de garder en mémoire leurs valeurs estimées et de modifier en fonction de leurs parentés, mère $\mathrm{j}$ et père $k$, les termes du système d'équations. On suppose ici ce fichier trié de telle façon que les parents y apparaissent avant leurs descendants.

\section{$\operatorname{VAL}(\mathrm{I})=$ GENE}

IF I. NE. O THEN DO

IF K. NE. O THEN DO

RHS $(J)=$ RHS $(J)+$ GENE*ALPHA-VAL $(K)^{*}$ ALPHA $/ 2$

$\mathrm{TAB}(\mathrm{J})=\mathrm{TAB}(\mathrm{J})+\mathrm{ALPHA} / 2$

END

ELSE DO

RHS $(J)=$ RHS $(J)+($ GENE*ALPHA $* 2) / 3$

$\mathrm{TAB}(\mathrm{J})=\mathrm{TAB}(\mathrm{J})+\mathrm{ALPHA} / 3$

END

END 
Ensuite, à la lecture d'un enregistrement du fichier des femelles, aux places i de l'individu, on ajoute aux termes de droite la quantité $r_{i}$ et aux éléments diagonaux $n_{i}$, et on sauvegarde dans MERE la codification $j$.

$$
\begin{aligned}
& \mathrm{A}=\mathrm{GAMA} /(\mathrm{N}+\mathrm{GAMA}) \\
& \text { TAB }(\mathrm{I})=\text { TAB }(\mathrm{I})+\mathrm{N}^{*} \mathrm{~A} \\
& \operatorname{RHS}(\mathrm{I})=\mathrm{RHS}(\mathrm{I})+\mathrm{R}^{*} \mathrm{~A} \\
& \operatorname{MERE}(\mathrm{I})=\mathrm{J}
\end{aligned}
$$

Dans le cas où les 2 parents sont identifiés, on doit ajouter à l'élément de droite de l'individu la valeur du père multipliée par $\alpha$ et soustraire au terme de droite de la mère la moitié de cette même quantité. Si seul le père est connu, alors on ajoute

seulement à la place de l'individu la valeur du père multipliée par $\frac{2}{3} \alpha$

IF K. NE. O THEN DO

IF J. NE O THEN DO

$\mathrm{A}=\mathrm{VAL}(\mathrm{K}) *$ ALPHA

RHS (I) $=$ RHS (I) + A

$\operatorname{RHS}(\mathrm{J})=\operatorname{RHS}(\mathrm{J})-\mathrm{A} / 2$

END

ELSE RHS (I) $=$ RHS $(\mathrm{I})+(2 * \operatorname{VAL}(\mathrm{K}) *$ ALPHA $) / 3$

END

Le calcul des termes de gauche, tableaux TAB et PAR se fait ensuite suivant les règles déjà décrites.

IF J. NE. O THEN DO

IF $K$. NE. O THEN A = ALPHA

ELSE $A=(2 *$ ALPHA $) / 3$

$\mathrm{D}=\mathrm{TAB}(\mathrm{I})+2 * \mathrm{~A}$

$\mathrm{TAB}(\mathrm{J})=\mathrm{TAB}(\mathrm{J})+\mathrm{A} / 2-\mathrm{A} * \mathrm{~A} / \mathrm{D}$

$\mathrm{D}=\mathrm{SQRT}(\mathrm{D})$

TAB $(\mathrm{I})=\mathrm{D}$

$\operatorname{PAR}(\mathrm{I})=-\mathrm{A} / \mathrm{D}$

END

ELSE DO

IF K. NE. O THEN TAB $(\mathrm{I})=$ TAB $(\mathrm{I})+(4 *$ ALPHA $) / 3$

END

ELSE TAB $(\mathrm{I})=$ TAB $(\mathrm{I})+$ ALPHA

Pour des raisons de clarté, l'organigramme recalcule pour chaque individu des quantités invariables fonctions de $\alpha$ et de $\mathrm{n}$ que l'on aura intérêt à calculer une seule fois à l'initialisation du programme.

\section{Résolution du système}

Le système $\mathrm{Lh}=\mathrm{r}$ se résout facilement grâce au stockage dans MERE de la codification de la mère de chaque individu. Pour chaque ligne $i$ on résoud $h_{i}=r_{i}$ et cette solution, stockée d'abord dans les termes de troite, est ensuite multipliée 
par le coefficient extradiagonal pour être soustraite du terme de droite correspondant à la mère.

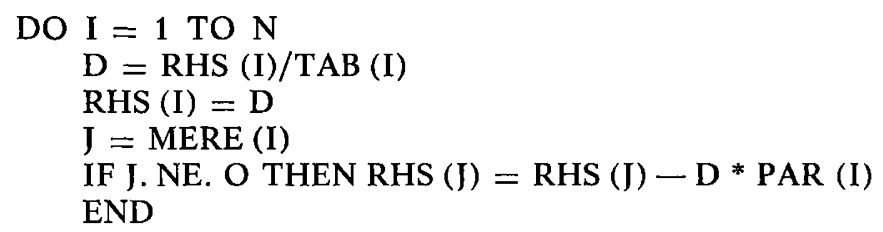

La résolution finale est enfin obtenue en parcourant les tableaux dans le sens inverse, en prenant soin de soustraire au terme de droite la solution déjà obtenue pour la mère multipliée par l'élément extradiagonal.

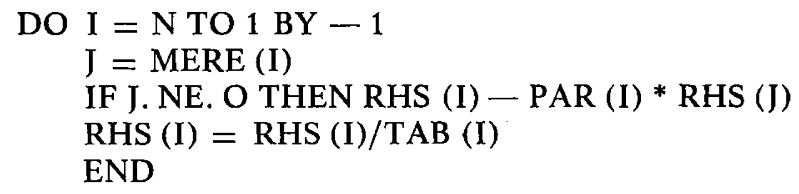

